\title{
A Computational Model of the Escape Response Latency in the Giant Fiber System of Drosophila melanogaster
}

\author{
${ }^{(1)}$ Hrvoje Augustin, ${ }^{1,2^{*}}$ Asaph Zylbertal, ${ }^{3^{*}}$ and Linda Partridge ${ }^{1,2}$
}

https://doi.org/10.1523/ENEURO.0423-18.2019

${ }^{1}$ Institute of Healthy Ageing, and GEE, University College London, London WC1E 6BT, United Kingdom, ${ }^{2}$ Max Planck Institute for Biology of Ageing, Cologne D-50931, Germany, and ${ }^{3}$ Department of Neuroscience, Physiology and Pharmacology, University College London, London WC1E 6BT, United Kingdom

\begin{abstract}
The giant fiber system (GFS) is a multi-component neuronal pathway mediating rapid escape response in the adult fruit-fly Drosophila melanogaster, usually in the face of a threatening visual stimulus. Two branches of the circuit promote the response by stimulating an escape jump followed by flight initiation. A recent work demonstrated an age-associated decline in the speed of signal propagation through the circuit, measured as the stimulus-to-muscle depolarization response latency. The decline is likely due to the diminishing number of inter-neuronal gap junctions in the GFS of ageing flies. In this work, we presented a realistic conductance-based, computational model of the GFS that recapitulates the experimental results and identifies some of the critical anatomical and physiological components governing the circuit's response latency. According to our model, anatomical properties of the GFS neurons have a stronger impact on the transmission than neuronal membrane conductance densities. The model provides testable predictions for the effect of experimental interventions on the circuit's performance in young and ageing flies.
\end{abstract}

Key words: aging; computational model; Drosophila; escape response; gap junctions; ion channels

\section{Significance Statement}

Finding potential targets for preventing functional decline of neuronal circuits is important from both biological and clinical perspective. In the nervous system of Drosophila melanogaster, the escape response system mediates quick propagation of signals from the brain to the muscles, instructing flight initiation following a threatening visual stimulus. It was previously shown that this circuit shows a marked decline in the speed of signal propagation with age, likely due to loss of synaptic gap junctions. Here, we generated a computational model of the system and uncovered novel anatomic and physiologic parameters that govern the circuit's function in young and old animals. These predictions can be tested experimentally and have significance for other fast circuits in flies and other species.

\section{Introduction}

Escape responses are evolutionarily ancient mechanisms used by many species as their main defense against predator attacks. Intense selection pressure has led to dedicated reflex circuits that continuously monitor the environment for danger and trigger escape behaviors when presented with a specific set of threatening stimuli. These circuits must be able to respond within a minimal time frame to prevent capture and maximize chances of survival (Herberholz et al., 2004; Walker et al., 2005). 


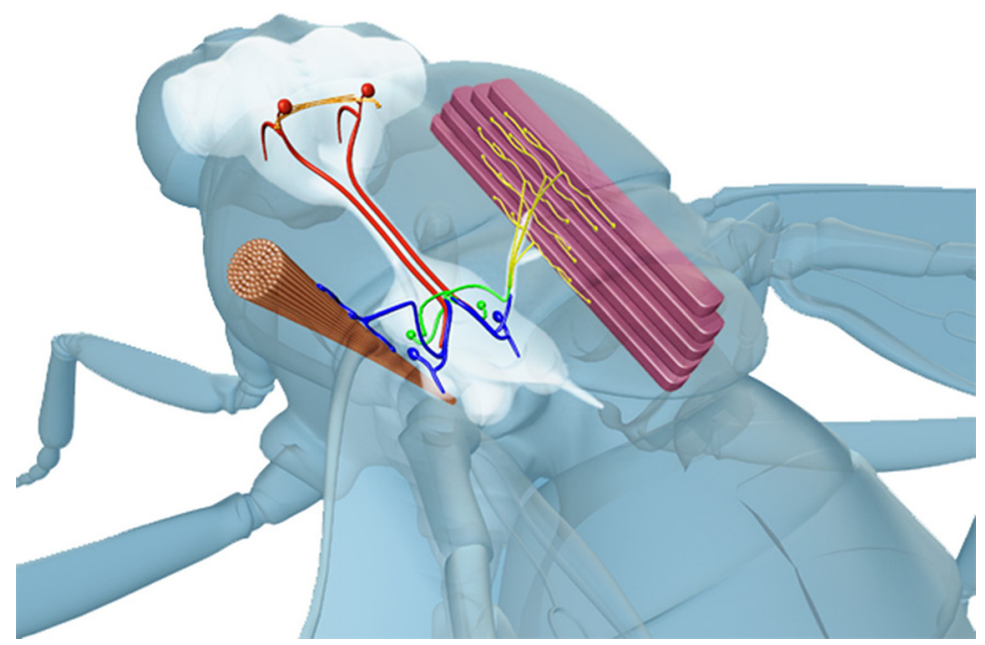

Figure 1. Diagram of the GFS anatomy. Two GF interneurons originating in the brain (red) descend to the thoracic ganglia where they connect, via a mixed (electrical and chemical) synapse, to the TTMn (blue) innervating the cylindrical TTM. In the second branch of the circuit, the GFs form a mixed synapse with the PSI (green), which, in turn, chemically synapses onto the DLMns (yellow) innervating the DLMs. Red circles in the brain denote approximate positions of the GF cell bodies.

Escape circuits are therefore characterized by extremely fast reaction times, with response latencies as short as a few milliseconds (Card and Dickinson, 2008; Dill, 1974). In dipteran insects, escape responses are mediated by the giant fiber system (GFS). Prompted by a visual (and, possibly, mechano-sensory) stimulus, the adult fruit-fly Drosophila melanogaster executes a stereotyped sequence of events that results in an escape jump followed by flight initiation (Trimarchi and Schneiderman, 1995; Allen et al., 2006; Fayyazuddin et al., 2006). The GFS consists of two descending, non-myelinated giant fiber (GF) interneurons that originate in the brain, and downstream neurons that innervate and activate flight muscles (dorsal longitudinal muscles, DLMs) and jump muscles (tergotrochanteral muscles, TTMs; King and Wyman, 1980; Sun and Wyman, 1997; Allen et al., 2006; Fig. 1). A single a single action potential (AP) in a GF axon is sufficient to initiate patterned activity in jump and flight muscles (Koto et al., 1981). Functionally, electrical synapses are a dominant type of synapse in the Drosophila GFS (Phelan et al., 1996; Trimarchi and Murphey, 1997), with chemical (cholinergic) synapses playing a minor role (Allen and Murphey, 2007). Gap junctions are the physical substrate of electrical synapses that provide physical continuity between the cytoplasms of closely apposed pre-

This work was funded by a Wellcome Trust Strategic Award to L.P. and by the Max Planck Society.

Acknowledgements: We thank Konstantinos Lagogiannis (King's College London) for providing useful comments on this manuscript, The Okinawa Institute of Science and Technology for organizing the Computational Neuroscience course where this project was initiated, and Marcus Allen for the artwork in Figure 1.

Correspondence should be addressed to Linda Partridge at linda.partridge@ucl.ac.uk

https://doi.org/10.1523/ENEURO.0423-18.2019

Copyright (C) 2019 Augustin et al.

This is an open-access article distributed under the terms of the Creative Commons Attribution 4.0 International license, which permits unrestricted use, distribution and reproduction in any medium provided that the original work is properly attributed. and post-synaptic neurons (Bennett, 1997). Compared to chemical synapses, transmission across electrical synapses is extraordinarily fast, with the possibility of the current flowing in either direction across the gap junction (Purves et al., 2001). Electrical synapses are therefore frequently found in places where fast transmission is critical, such as in escape response and motion-processing circuits (Cook and Becker, 1995). In the Drosophila GFS, the shaking-B gene (shakB, in $x 8$ ) instructs the formation of heterotypic, unidirectional (rectifying) electrical synapses (Phelan et al., 1998; Stebbings et al., 2002; Wu et al., 2011).

Loss of gap junctions in the nervous system occurs normally as a consequence of aging. For example, astrocytic gap junctional plaques are drastically reduced in the brains of aging mice (Cotrina et al., 2001), likely affecting inter-astrocytic and neuron-glia metabolic coupling (Cai et al., 2017). The structural proteins comprising the gap junctional channels are called connexins or pannexins in vertebrates (Hormuzdi et al., 2004) and innexins in invertebrate species (Hasegawa and Turnbull, 2014). In various knock-out mutants, widespread disruption of the neuronal gap junctional coupling leads to reduced synchronicity of neural networks (Deans et al., 2001), impaired oscillatory patterns in the brain (Buhl et al., 2003), neuronal hyperexcitability (Sutor et al., 2000), increased neuronal apoptosis (Nakase et al., 2003), and reduced neuroprotection after ischemic injury (Siushansian et al., 2001).

Recently, Augustin et al. (2017) showed that the response latency through the GFS (i.e., the time between the stimulation of the GFs in the brain, and flight or jump muscle depolarization) increases with age, demonstrating an age-related decline in the functionality of the escape circuit. These experimental results suggest that the prolonged signal propagation is likely due to the ageassociated decline in the conductance via gap junctions. This hypothesis is based on the findings that the old flies exhibited severely reduced ShakB plaque size (indicating 
A

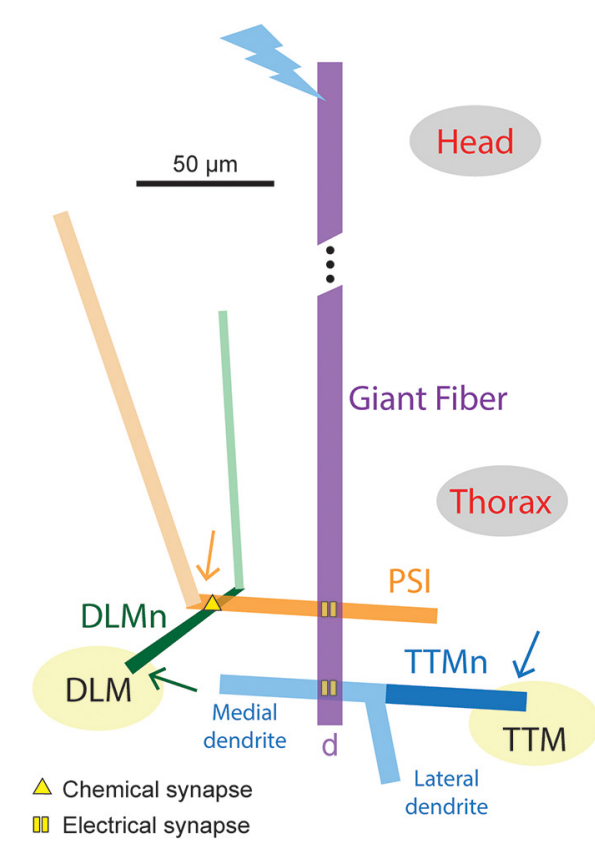

B

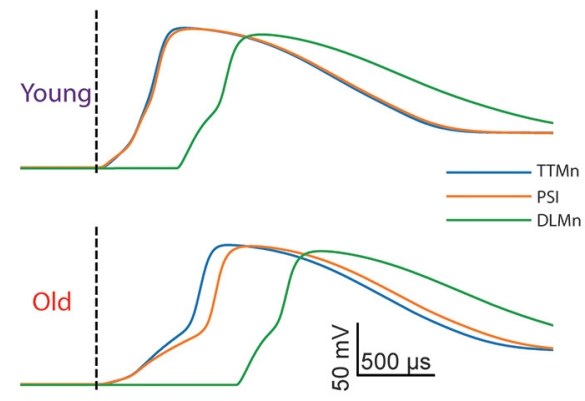

C

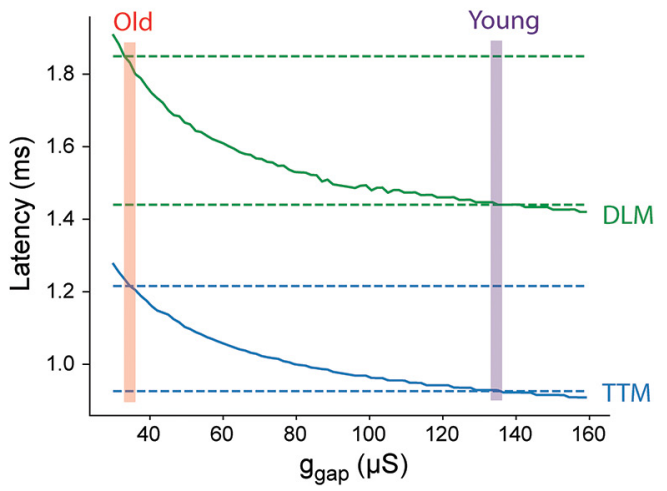

Figure 2. GFS model architecture and response latency measurements. A, Model architecture and geometry, showing the cylindrical sections that make up the four cell types in the model (to scale), along with the location of electrical and chemical synapses. Active sections (axons) are shown in dark colors. Bolt denotes the proximal end of the GF that is stimulated in the simulation, and arrows denote the distal ends of the axons, from which the voltage recordings shown in $\boldsymbol{B}$ were taken. The response latency in the DLM pathway is slightly delayed compared to the latency in the (shorter) TTM branch. $\boldsymbol{B}$, Membrane potential recorded in the model TTMn (blue), DLMn (green), and PSI (orange), for "young fly" $g_{\text {gap }}$ value (135 $\mu \mathrm{S}$, top) and "old fly" g gap value (34.5 $\mu \mathrm{S}$, bottom). $\boldsymbol{C}, \mathrm{Latency}$ from stimulus onset to muscle response as predicted by the model for TTM (blue) and DLM (green), as a function of $g_{g a p}$. The latency values recorded experimentally are indicated by dashed lines, and the $g_{\text {gap }}$ values where they coincide with the values predicted by the model are shown by magenta and red bars (for young and old flies, respectively).

reduced gap junctional volume and consequent attenuation of the junctional conductance), with other potentially contributing factors to this decline such as neuromuscular function and GF diameter being unaffected by age (Augustin et al., 2017). In this study, we generated a realistic computational biophysical model of the GFS based on these findings and on previously reported properties of the circuit's components. By exploring potential determinants of response latency, including membrane properties, neuronal geometry and gap junction conductance, we created a model that not only recapitulates the previously reported experimental results, but also elucidates the relative importance of different physiologic and anatomic parameters in regulating the speed of signal propagation through this escape response circuit.

\section{Materials and Methods}

\section{Code accessibility}

The code described in the paper is freely available online at http://modeldb.yale.edu/245415. The code is available as Extended Data 1.

\section{Model architecture}

To implement the model, we used the NEURON simulation environment with Python (Hines and Carnevale, 1997; Hines et al., 2009) ran on a Dell PC laptop using
Ubuntu operating system. The model of the Drosophila GFS (of either sex) is comprised of four cells: The GF neuron, the TTM motoneuron (TTMn), a peripherally synapsing interneuron (PSI), and a DLM motoneuron (DLMn; Fig. 2A). Each neuron contains one to three unbranched cylindrical sections (functional subunits) with dimensions based on anatomic data (see model parameters below).

Each section is divided into 51 iso-potential segments, that form the basic computational unit of the model and connected via fixed specific axial resistance. The membrane potential in each segment is calculated as a function of time based on the cable equation and any fixed or time-varying membranal conductances it contains. The GF is modeled as a single active section that forms unidirectional electrical synapses onto the active section (axon) of the PSI and the medial passive section (dendrite) of the TTMn. The TTMn contains two dendrites (medial and lateral; Godenschwege et al., 2002a) and an active axon. The PSI contains a dendrite and an axon, which forms a chemical synapse onto the active section (axon) of the DLMn. The DLMn contains a tapering axon and a dendrite (King and Wyman, 1980; Egger et al., 1997).

\section{Model conductances}

All model sections contain a passive leak conductance. Active sections (axons) were largely modeled 
Table 1. Anatomic and physiologic parameters used in the article

GF diameter

GF length

Distance of contact with TTMn from proximal end

Distance of contact with PSI from proximal end

TTMn diameter

TTMn axon length

TTMn medial dendrite length

TTMn lateral dendrite length

Distance of input from GF from medial dendrite proximal end

PSI diameter

PSI axon length

PSI dendrite length

Distance of input from GF from PSI axon proximal end

Distance of contact with DLMn from PSI axon proximal end

DLMn dendrite and axon proximal diameter

DLMn axon distal diameter

DLMn axon length

DLMn dendrite length

Distance of input from PSI from axon proximal end

Physiological parameters

Leak conductance

Specific membrane capacitance

Specific axial resistance

Maximal transient voltage-gated sodium conductance $\left(\bar{g}_{\mathrm{Nat}}\right)$

Maximal persistent voltage-gated sodium conductance $\left(\bar{g}_{\text {Nap }}\right)$

Maximal voltage-gated potassium conductance $\left(\bar{g}_{K}\right)$

Gap junctions conductance ( $g_{\text {gap }}$, young fly)

Gap junctions conductance ( $g_{\text {gap }}$, old fly)

Chemical synapse rise $\tau$

Chemical synapse decay $\tau$

Chemical synapse reversal potential

Chemical synapse delay

Chemical synapse peak conductance

Neuromuscular junction delay

Leak reversal potential

Sodium reversal potential

Potassium reversal potential
Anatomical parameters

$8 \mu \mathrm{m}$ (Augustin et al., 2017)

$400 \mu \mathrm{m}$ (Phelan et al., 1996; Smith et al., 1996)

$400 \mu \mathrm{m}$ (distal end of the GF)

$360 \mu \mathrm{m}$ (King and Wyman, 1980; Phelan et al., 1996)

$6 \mu \mathrm{m}$ (King and Wyman, 1980)

$50 \mu \mathrm{m}$ (Godenschwege et al., 2002b)

$60 \mu \mathrm{m}$ (Godenschwege et al., 2002b)

$30 \mu \mathrm{m}$ (Godenschwege et al., 2002b)

$12 \mu \mathrm{m}$ (Godenschwege et al., 2002a,b)

$4.5 \mu \mathrm{m}$ (King and Wyman, 1980)

$90 \mu \mathrm{m}$ (Phelan et al., 1996; Egger et al., 1997)

$170 \mu \mathrm{m}$ (estimated)

$45 \mu \mathrm{m}$ (Egger et al., 1997; Blagburn et al., 1999)

$76.5 \mu \mathrm{m}$ (Egger et al., 1997)

$2 \mu \mathrm{m}$ (King and Wyman, 1980)

$4 \mu \mathrm{m}$ (King and Wyman, 1980)

$50 \mu \mathrm{m}$ (Sun and Wyman, 1997)

$100 \mu \mathrm{m}$ (Sun and Wyman, 1997)

$12.5 \mu \mathrm{m}$ (Egger et al., 1997)

$0.03 \mathrm{mS} / \mathrm{cm}^{2}$ (estimated)

$1 \mu \mathrm{F} / \mathrm{cm}^{2}$ (estimated)

$35.4 \Omega / \mathrm{cm}$ (estimated)

$300 \mathrm{mS} / \mathrm{cm}^{2}$ (Günay et al., 2015)

$0.11 \mathrm{mS} / \mathrm{cm}^{2}$ (Günay et al., 2015)

$10 \mathrm{mS} / \mathrm{cm}^{2}$ (estimated)

$135 \mu \mathrm{S}$ (estimated)

$34.5 \mu \mathrm{S}$ (estimated)

$0.1 \mathrm{~ms}$ (standard value)

$1 \mathrm{~ms}$ (standard value)

0 (standard value)

$0.15 \mathrm{~ms}$ (estimated)

$80 \mu \mathrm{S}$ (estimated)

$0.35 \mathrm{~ms}$ (see Materials and Methods)

$-85 \mathrm{mV}$ (Günay et al., 2015)

$65 \mathrm{mV}$ (Günay et al., 2015)

-74 mV (Günay et al., 2015) according to an existing model of a Drosophila motoneuron (Günay et al., 2015) and based on HodgkinHuxley type channel kinetics (Hodgkin and Huxley, 1952). They contain persistent and transient voltagegated sodium channels, as well as voltage-gated potassium channels, with kinetics based on Günay et al. (2015). Each conductance type is distributed with equal density in all active sections. The PSI-DLMn chemical synapse is modeled as a double-exponential process.

\section{Simulation}

To test the TTM and DLM response latency in the model, we stimulated the proximal end of the GF with a current step duration of $0.03 \mathrm{~ms}$ (similar to that used by Augustin et al., 2017) and amplitude of 120 nA to approximate the input to the GF during high-amplitude head stimulation and measured the latency to the AP peak in the TTMn and DLMn. To compare this latency with the latency values measured experimentally, we added 0.35 $\mathrm{ms}$ to this value to account for the neuromuscular junction delay. This value was estimated from the experimentally measured "neuromuscular latency" of $\sim 0.65 \mathrm{~ms}$. The neuromuscular latency is the time period between tho- racic stimulation that directly stimulates the motoneurons, and TTM or DLM (muscle) depolarization (Augustin et al., 2017). This duration is the sum of two periods: (1) the time that passes from thoracic stimulus onset to AP peak in the TTMn or DLMn, and (2) the time from the AP peak to muscle depolarization (NMJ delay). To estimate the first part, the model TTMn was stimulated directly (simulating thoracic stimulation), resulting in $\sim 0.3 \mathrm{~ms}$ from stimulus onset to AP peak. The estimated NMJ delay is therefore the remaining $0.35 \mathrm{~ms}$, achieving a total of $0.65 \mathrm{~ms}$. This delay contributes a fixed bias to the latency values, and therefore plays no role in assessing the relative importance of model parameters.

\section{Model parameters}

The model parameters were chosen according to known values from the literature, where available (Table 1). Some of these values were manually adjusted to make sure all the model cells are spiking, and the response latencies match recorded values. Dimensions in the simplified anatomy were chosen to capture the general proportions of the cells and the ratio between active and passive membrane area. 
A
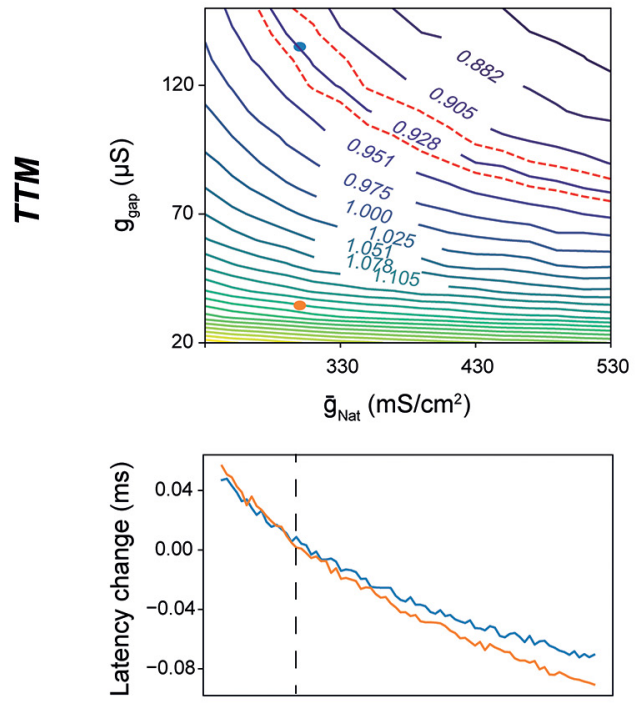

D
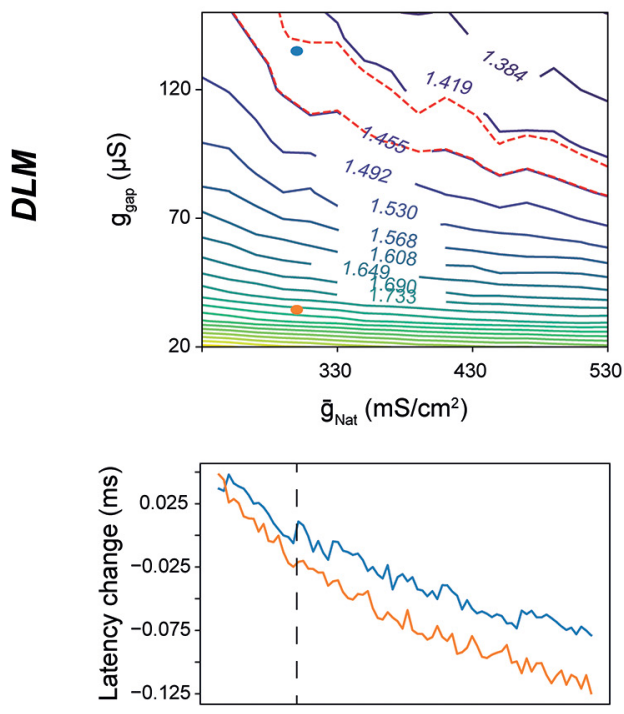

B
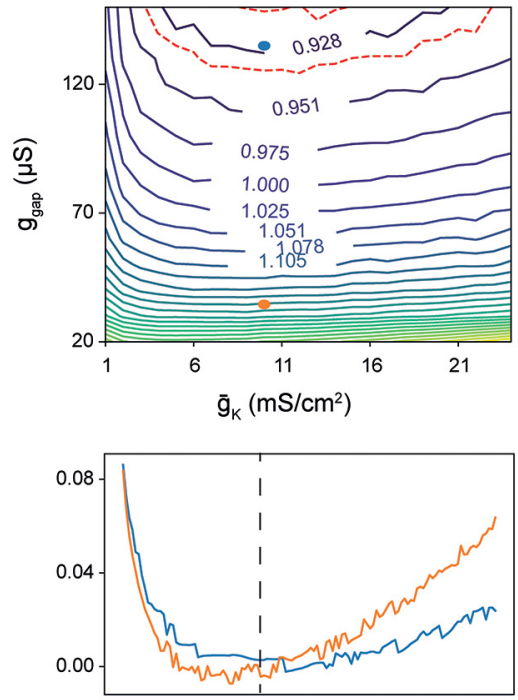

E
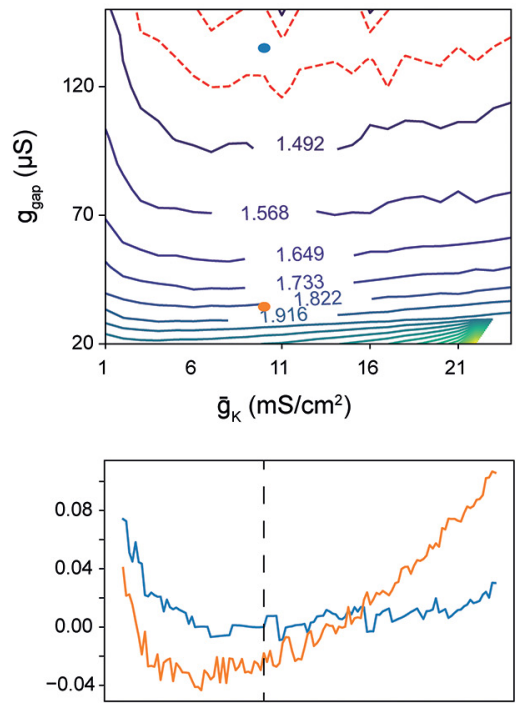

C
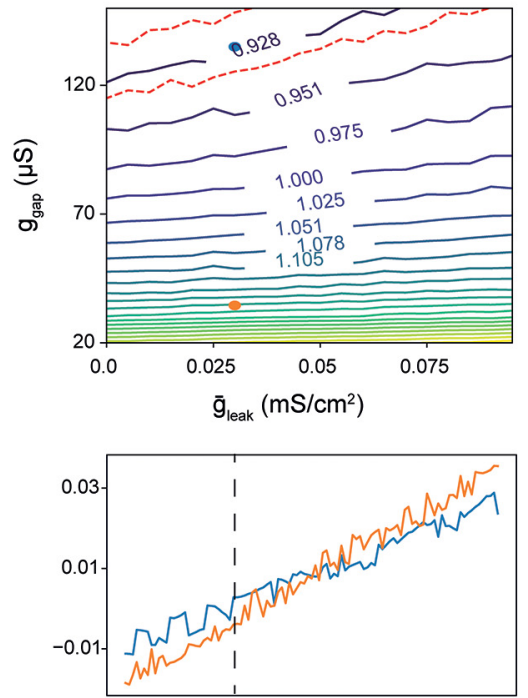

F

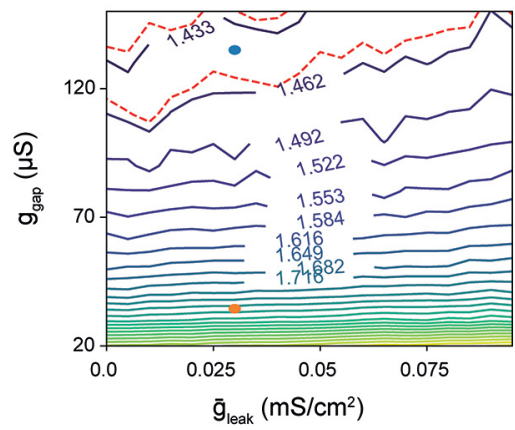

-

Figure 3. Co-dependency of the response latency on $\mathrm{g}_{\mathrm{gap}}$. $\boldsymbol{A}-\boldsymbol{C}$, top, The latency landscape in the TTM, shown using iso-latency lines (labeled with response latency values in milliseconds) as a function of the global gap junction conductance $\left(g_{\mathrm{gap}}\right)$ and maximal transient voltage-gated sodium conductance $\left(\mathrm{g}_{\mathrm{Nat}}^{-}, \boldsymbol{A}\right)$, maximal voltage-gated potassium conductance $\left(\mathrm{g}_{\mathrm{K}}^{-}, \boldsymbol{B}\right)$, and leak conductance $\left(\mathrm{g}_{\text {leak }}^{-}, \boldsymbol{C}\right)$. Blue and orange dots represent the values for young and old flies, respectively. The region in the landscape representing young fly latency is marked by red dashed lines. Bottom, Cross sections in the latency landscape, showing the change in latency (relative to experimentally measured values) as a function of the three conductance types, for young flies (blue) and old flies (orange). $\boldsymbol{D}-\boldsymbol{F}$, same as $\boldsymbol{A}-\boldsymbol{C}$, for the DLM.

\section{Results}

\section{A conductance-based model of the Drosophila GFS reproduces aging-related latency increase}

To examine how the electrical coupling in the fly's GFS contributes to the transmission latency, we stimulated the model circuit with a 120-nA pulse at the proximal end of the GF (Fig. $2 A$ ) and recorded the voltage at the distal ends of the TTMn, PSI, and DLMn neurons. Setting the gap junction conductance $\left(g_{\text {gap }}\right)$ of all model electrical synapses to $135 \mu \mathrm{S}$ resulted in the voltage recordings shown in Figure $2 B$, top. The latency from stimulus onset to AP peak, summed up with a fixed neuromuscular junction latency $(0.35 \mathrm{~ms})$, matches the values recorded experimentally in the TTM and DLM of young (5-7 d old) flies (0.93 and $1.44 \mathrm{~ms}$, respectively; Augustin et al., 2017). This value of $g_{\text {gap }}$ will therefore be used to model the response latency in young flies. When using this value, the latency in the PSI and TTMn cells is similar, owing to their equivalent position in the circuit (one electrical synapse away from the GF).

Decreasing $g_{\text {gap }}$ to $34.5 \mu \mathrm{S}$ results in longer membrane charging time to firing threshold due to weaker current 
A

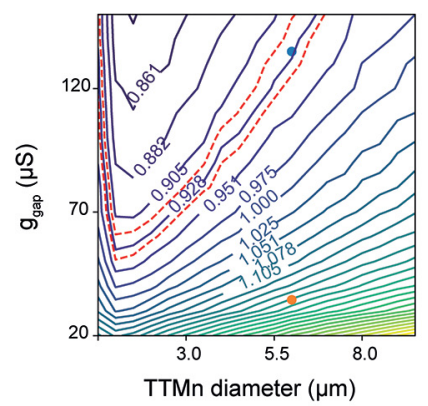

E

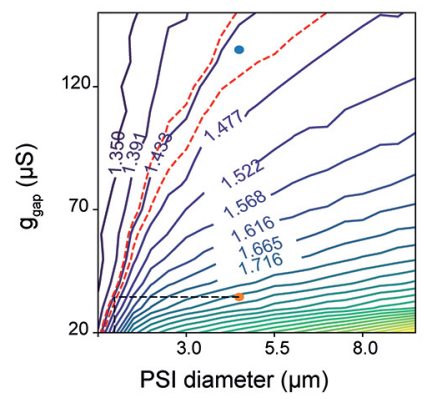

B

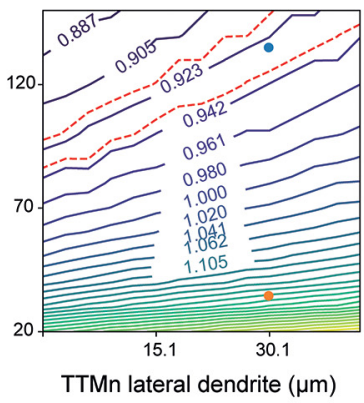

F

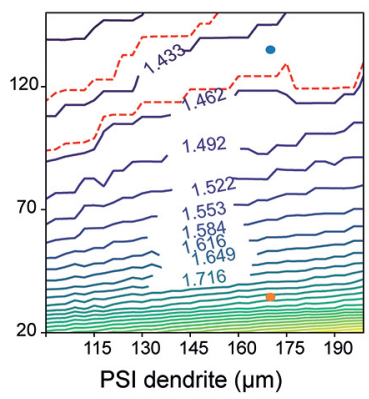

C

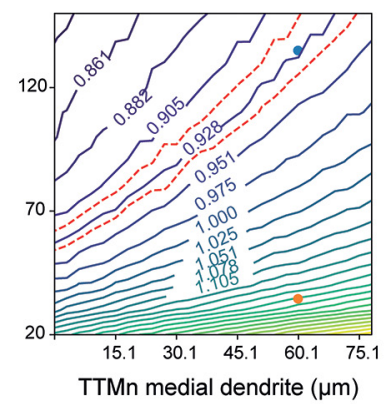

G

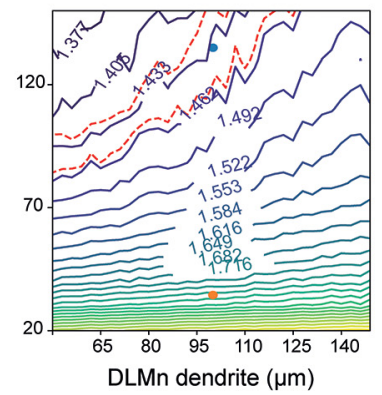

D

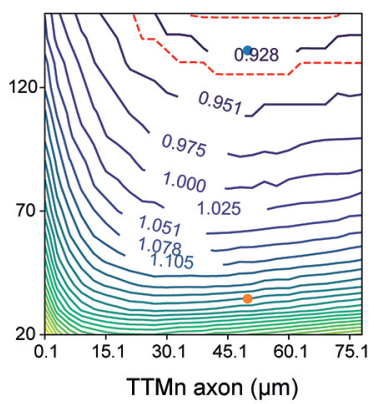

H

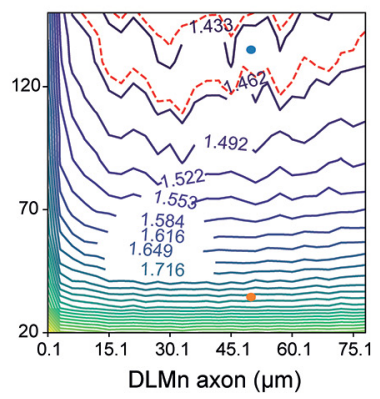

Figure 4. Impact of anatomic model parameters on response latency. $\boldsymbol{A}-\boldsymbol{D}$, TTM Latency as a function of $g_{g a p}$ and anatomic parameters in the TTM branch of the model: the diameter of TTMn sections $(\boldsymbol{A})$, and the length of the TTMn lateral dendrite $(\boldsymbol{B})$, medial dendrite $(\boldsymbol{C})$, and axon $(\boldsymbol{D})$. $\boldsymbol{E}-\boldsymbol{H}$, DLM latency as a function of $\mathrm{g}_{\mathrm{gap}}$ and anatomic parameters in the DLM branch of the model: PSI section diameter $(\boldsymbol{E})$, PSI dendrite length $(\boldsymbol{F})$, DLMn dendrite length $(\boldsymbol{G})$, and DLMn axon length $(\boldsymbol{H})$.

across the gap junction, and thus to increased latency for both TTM and DLM, up to the values recorded in old (45-50 d old) flies (1.22 and $1.85 \mathrm{~ms}$; Fig. 2B, bottom). This value of $g_{\text {gap }}$ will therefore be used to model the response latency in old flies. When using this value, the latency in the PSI becomes larger compared to the TTMn, since at lower inward currents the differences in morphology between these cells play a bigger role (namely, the long dendrite of the PSI that increases the load on the input current). Scanning a range of conductance values reveals the expected monotonic decrease of the response latency as $\mathrm{g}_{\mathrm{gap}}$ increases (Fig. 2C). The model therefore reproduces the time latencies from stimulus to jump and flight muscle depolarization and shows that a 4-fold reduction in gap junction conductance by itself could account for the transition from the latencies measured in young flies to the latencies measured in old flies.

\section{Co-dependency of the response latency on $\mathbf{g}_{\text {gap }}$ and other physiologic and anatomic parameters}

Next, we tested how the response latency predicted by the model changes as a function of both $g_{\text {gap }}$ and membranal conductance densities. We performed two-dimensional grid scans by varying $g_{\text {gap }}$ (spanning the values used for young and old flies), along with either the maximal transient voltage-gated sodium conductance for all axons $\left(\bar{g}_{\mathrm{Nat}}\right.$; Fig. $\left.3 A, D\right)$, the maximal voltage-gated potassium conductance for all axons $\left(\bar{g}_{\mathrm{K}} ;\right.$ Fig. $\left.3 B, E\right)$, or the leak conductance in all sections $\left(g_{\text {leak }}\right.$; Fig. $\left.3 C, F\right)$. The latency values are presented as contour maps, where regions in the parameter space with a similar latency as young flies ( $\pm 1 \%$ ) are denoted by red dashed lines. For example, Figure $3 A$, top, shows that similar TTM response latency as that of young flies $(0.928 \mathrm{~ms})$ is achieved when a reduction in $\mathrm{g}_{\text {gap }}$ (moving toward the bottom) is compensated by an increase in $\bar{g}_{\text {Nat }}$ (moving toward the right). The plots below the contour maps show the change in latency for young (blue) and old (orange) flies as a function of the conductance in question, thus they represent horizontal cross sections through the contour maps. While the directionalities of the effects on response latency were expected from basic biophysical principles, this approach enabled us to assess the relative efficacies of these changes. As expected, increased transient voltage-gated sodium conductance reduced the response latencies (Fig. $3 A, D)$ due to lowered AP threshold, and to a lesser degree by faster AP propagation. The reverse effect was observed when increasing the potassium and leak conductances (Fig. 3B,C,E,F), since these changes shift the membrane potential away from firing threshold and shunt inward current, leading to an increase in the time needed to reach threshold during a pre-synaptic spike. An extreme reduction in potassium conductance also prolonged the latency (Fig. $3 B, E$, left side of the plot) by elevating the resting membrane potential and thus causing sodium conductance inactivation. Within the tested ranges of conductance values, no change in a single parameter reverted the latency of an old fly (orange dot) to that of a young fly. 
A
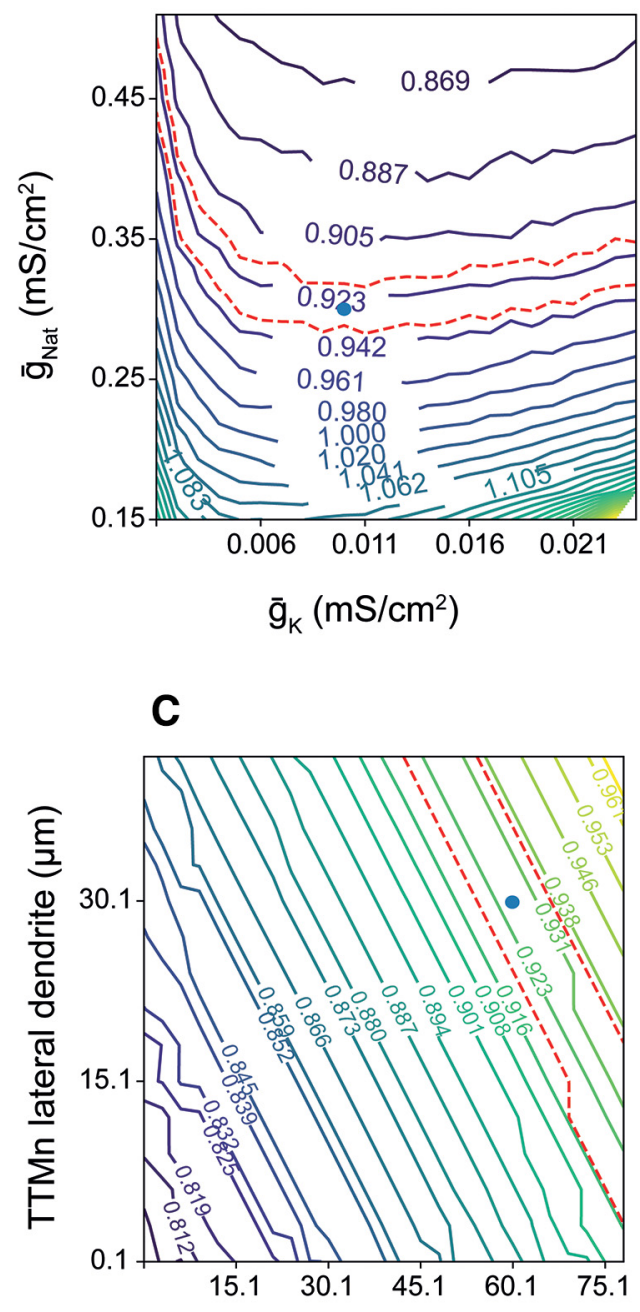

TTMn medial dendrite $(\mu \mathrm{m})$
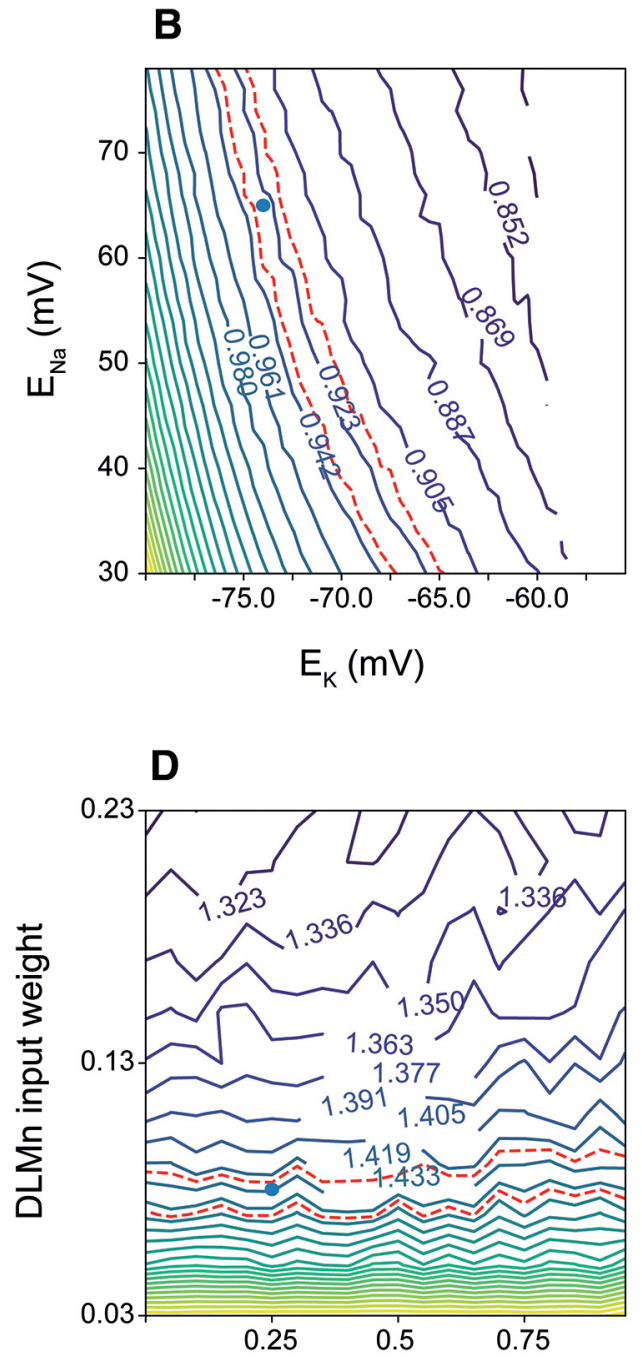

DLMn input location

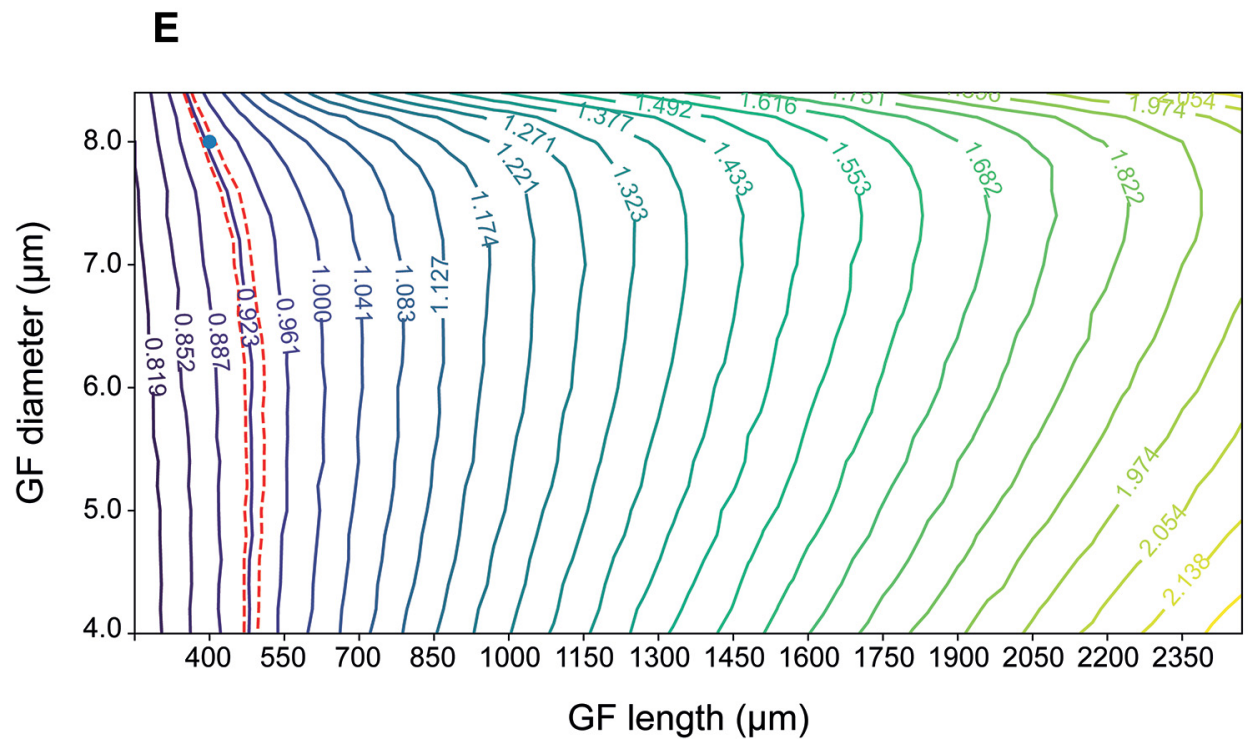

Figure 5. Co-dependency of the response latency on different parameter combinations. $\boldsymbol{A}$, TTM latency as a function of maximal 
continued

voltage-gated transient sodium conductance $\left(\mathrm{g}_{\mathrm{Nat}}^{-}\right)$and maximal voltage-gated potassium conductance $\left(\mathrm{g}_{\mathrm{K}}^{-}\right)$. B, TTM latency as a function of sodium reversal potential $\left(E_{\mathrm{Na}}\right)$ and potassium reversal potential $\left(\mathrm{E}_{\mathrm{K}}\right)$. C, TTM latency as a function of the TTMn medial dendrite length and TTM lateral dendrite length. $\boldsymbol{D}$, DLM latency as a function of the PSI-to-DLMn chemical synapse weight, and the synapse location along the DLMn dendrite. $\boldsymbol{E}$, GF latency as a function of the GF diameter and length. Blue dots represent the values for young and old flies, respectively.

We next tested how the response latency varied when changing $g_{\text {gap }}$ along with anatomic parameters. For the TTMn branch of the circuit, we tested the following parameters: TTMn diameter (Fig. $4 A$ ), the length of lateral and medial TTMn dendrites (Fig. 4B,C), and the TTMn axon length (Fig. 4D). For the DLMn branch, we tested the PSI diameter (Fig. 4E), PSI dendrite length (Fig. 4F), DLMn dendrite length (Fig. 4G), and the DLMn axon length (Fig. $4 H)$. Since increases in diameter and length of neuronal sections decrease the cells' input resistance, these changes in general decreased the effect of a given input current on the membrane potential and thus prolonged the latency to response. Among the tested parameters, a $\sim 5$-fold decrease in the PSI diameter reverted the response latency of an old fly to that of a young fly (Fig. $4 E$, black dashed line). Contrary to the general trend, where a reduction of section dimensions reduces the latency, a reduction in axonal length beyond a critical value prolonged the response latency due to the decrease in active membrane surface area (and thus, in total active conductance, Fig. 4D,H). Overall, changes in the diameter of neuronal section affected the membrane capacitance throughout the length of the section, making their influence on the latency stronger compared to changes in section length. The results from Figures 3, 4 show that, according to the model, changes in membrane conductance densities (within realistic limits) are far less efficient compared to anatomic changes in affecting the response latencies through the two branches of the GF circuit.

\section{Co-dependency of the response latency on other parameter combinations}

Our model enables predictions of the response latency as a function of arbitrary parameter combinations and may therefore be used as a benchside tool in experimental studies of signal propagation via the GFS. For example, Figure $5 \mathrm{~A}$ shows the TTM latency landscape for voltage-gated sodium and potassium maximal conductances, each affecting the latency in a similar way as shown earlier against $g_{\text {gap }}$ (Fig. $3 A, B$ ). Sodium and potassium reversal potentials, functions of intracellular and extracellular ionic concentrations, influence the resting membrane potential, and thus their depolarization reduces the latency to TTM response (Fig. 5B). Increase in the TTMn medial and lateral dendrite lengths leads to an increase in membrane load and thus to increased TTM latency (Fig. $5 \mathrm{C}$, see also Fig. $4 A-H$ ), but the medial dendrite length is more influential, since the GFs form synapses with the TTMn on this dendrite (Godenschwege et al., 2002a). The PSI-to-motoneuron contacts are chemical (Tanouye and Wyman, 1980; Allen and Murphey, 2007), so increasing the weight of this synapse naturally shortens the DLM response latency by accelerating the formation of AP in the DLMn as a response to PSI activity; the synapse location on the DLMn dendrite has hardly any influence (Fig. 5D). We also tested the impact of the GF length and diameter on response latency through the GFS (Fig. 5E). For shorter axons (left side), the optimal diameter (where minimal latency is achieved) is rather small, since the longitudinal AP propagation time is negligible compared to the passive charging of the membrane (see also Fig. $4 A, E$ ). For longer axons (right side), the propagation time becomes more important compared to membrane charging, so the optimal diameter is larger.

In summary, among the tested parameter combinations we identified potassium reversal potential and dendrite length as the parameters with high impact on response latency, with the DLMn input location having a relatively small effect. These results can help guide experiments aimed at manipulating the GFS latency responses.

\section{Discussion}

Our biophysical model of the Drosophila GFS accomplished three main things. Firstly, it recapitulated the latency responses previously measured in young and old flies (Augustin et al., 2017). The so called "short-latency responses" are elicited by applying a high-frequency stimulus to the brain, thereby directly activating the GF interneurons and bypassing the presynaptic (sensory) inputs to the GFs (Tanouye and Wyman, 1980; Engel and $\mathrm{Wu}$, 1996). These "stimulus-to-muscle depolarization" response times therefore represent a readout for the functionality of the GFS that includes the GFs, the interneurons and motoneurons downstream of the GFs, as well as the (jump and flight) muscles innervated by the motoneurons (Fig. 1). As the neuromuscular latency is not compromised by age (Augustin et al., 2017), we excluded the muscles (and their respective neuromuscular junctions) from the model, focusing on the period between the brain stimulus and motoneuronal (TTMn and DLMn) AP peak. To be fully explained solely by the properties of gap junctions, our model suggests that the experimentally measured age-related increase in response latency requires a $\sim 75 \%$ reduction in gap junctional conductance (Fig. 2B,C). While decreased conductance can be caused by either gap junctional loss or dysfunction, this level of attenuation of conductance via gap junctions is within the estimate of the age-associated gap junctional loss in the GFS (Augustin et al., 2017).

Secondly, our model demonstrated the degree to which manipulations of principal membrane ionic conductances can influence the GFS response latencies. Augmentation of the transient voltage-gated sodium conductance in our model (equivalent to an overexpression or hyperactivation of voltage-gated sodium channels in vivo) shortened the response latency, with the increase in potassium and leak 
currents having the expected, opposite, effect (Figs. 3, $5 A$ ). Considering the critical role of sodium currents in the generation of APs (Elmslie, 2010), the effect of increased $\mathrm{Na}^{+}$conductance is likely due to lowered AP threshold. However, when manipulated within a physiologically relevant range, sodium conductances were unable to revert the latencies from old flies to youthful levels (Fig. $3 A, D$ ).

Thirdly, we showed that anatomic features of the GFS neurons can have a stronger effect on the speed of signal propagation via the circuit (Figs. 4, 5C) compared to physiologic parameters. Since the main factor in the model's response latency is the membrane charging time to AP threshold, any change to the membranal load (e.g., change in membrane surface area as a consequence of changing the length or diameter of a neuronal section) strongly affects the response latency by modulating the cells' response dynamics to input current. Interestingly, decreasing the diameter of the PSI in old flies resulted in the reversal of response latencies to youthful values. For longer axons such as the GF, however, the propagation velocity may be more important than the membrane charging time (which is influenced by the membranal load; Rall, 2011). Indeed, optimal GF axon diameters scale with the length of the axonal section in determining the GFS response latency such that for short fibers the diameter that produces the minimal latency is smaller than the one for long fibers (Fig. 5E). The wide range of values for these anatomic and physiologic parameters shown in the contour maps does not necessarily represent values that are present or measured in vivo; it does, however, make it possible to determine, for example, whether the effect of various physiologic and morphologic parameters is continuous or whether there is a threshold beyond which an effect occurs.

Since the bulk of the signal propagation in the circuit is done via long axons and with minimal convergence of inputs, we could use simple, reduced geometry to represent the circuit components. With the addition of ion channel parameters previously characterized in Drosophila motoneuron simulations and several literature-based physiologic constraints, our model is likely to faithfully reproduce salient anatomic and physiologic features of the GFS.

The versatile Drosophila genetic toolbox allows for time-controlled modulation of relevant membrane conductances in individual GFS neurons and in the whole circuit (Olsen and Wilson, 2008; Jenett et al., 2012). Genetic manipulations of neuronal morphologies, including the size dendritic and axonal sections, are also possible (Scott et al., 2003; Sugimura et al., 2004), although these interventions have not yet been tested in the GFS. These tools can be used to experimentally test the results presented here, with the goal of increasing signal propagation speed through the circuit and furthering our understanding of this escape response system. Although our model is only partially constrained and some of its parameters had to be estimated, it is useful in developing intuition on which circuit elements are likely to have greater influence on the GFS response latency. The model code was made available and can be easily adapted to explore additional parameter combinations in addition to the ones presented here, and updated as more constraints become available.

\section{References}

Allen MJ, Murphey RK (2007) The chemical component of the mixed GF-TTMn synapse in Drosophila melanogaster uses acetylcholine as its neurotransmitter. Eur J Neurosci 26:439-445.

Allen MJ, Godenschwege TA, Tanouye MA, Phelan P (2006) Making an escape: development and function of the Drosophila giant fibre system. Semin Cell Dev Biol 17:31-41.

Augustin H, McGourty K, Allen MJ, Madem SK, Adcott J, Kerr F, Wong CT, Vincent A, Godenschwege T, Boucrot E, Partridge L (2017) Reduced insulin signaling maintains electrical transmission in a neural circuit in aging flies. PLoS Biol 15:e2001655.

Bennett MV (1997) Gap junctions as electrical synapses. J Neurocytol 26:349-366.

Blagburn JM, Alexopoulos H, Davies JA, Bacon JP (1999) Null mutation in shaking-B eliminates electrical, but not chemical, synapses in the Drosophila giant fiber system: a structural study. J Comp Neurol 404:449-458.

Buhl DL, Harris KD, Hormuzdi SG, Monyer H, Buzsáki G (2003) Selective impairment of hippocampal gamma oscillations in connexin-36 knock-out mouse in vivo. J Neurosci 23:1013-1018.

Cai W, Zhang K, Li P, Zhu L, Xu J, Yang B, Hu X, Lu Z, Chen J (2017) Dysfunction of the neurovascular unit in ischemic stroke and neurodegenerative diseases: an aging effect. Ageing Res Rev 34:77-87.

Card G, Dickinson MH (2008) Visually mediated motor planning in the escape response of Drosophila. Curr Biol 18:1300-1307.

Cook JE, Becker DL (1995) Gap junctions in the vertebrate retina. Microsc Res Tech 31:408-419.

Cotrina ML, Gao Q, Lin JH, Nedergaard M (2001) Expression and function of astrocytic gap junctions in aging. Brain research 90: $55-61$.

Deans MR, Gibson JR, Sellitto C, Connors BW, Paul DL (2001) Synchronous activity of inhibitory networks in neocortex requires electrical synapses containing connexin36. Neuron 31:477-485.

Dill ML (1974) The escape response of the zebra danio (Brachydanio rerio) I. The stimulus for escape. Anim Behav 22:711-722.

Egger MD, Nowakowski RS, Peng B, Wyman RJ (1997) Patterns of connectivity in a Drosophila nerve. J Comp Neurol 387:63-72.

Elmslie KS (2010) Action potential: ionic mechanisms. In: Encyclopedia of life sciences. Chichester, United Kingdom: John Wiley \& Sons Ltd.

Engel JE, Wu CF (1996) Altered habituation of an identified escape circuit in Drosophila memory mutants. J Neurosci 16:3486-3499.

Fayyazuddin A, Zaheer MA, Hiesinger PR, Bellen HJ (2006) The nicotinic acetylcholine receptor Dalpha7 is required for an escape behavior in Drosophila. PLoS Biol 4:e63.

Godenschwege TA, Hu H, Shan-Crofts X, Goodman CS, Murphey RK (2002a) Bi-directional signaling by Semaphorin 1a during central synapse formation in Drosophila. Nat Neurosci 5:1294-1301.

Godenschwege TA, Simpson JH, Shan X, Bashaw GJ, Goodman CS, Murphey RK (2002b) Ectopic expression in the giant fiber system of Drosophila reveals distinct roles for roundabout (Robo), Robo2, and Robo3 in dendritic guidance and synaptic connectivity. J Neurosci 22:3117-3129.

Günay C, Sieling FH, Dharmar L, Lin WH, Wolfram V, Marley R, Baines RA, Prinz AA (2015) Distal spike initiation zone location estimation by morphological simulation of ionic current filtering demonstrated in a novel model of an identified Drosophila motoneuron. PLoS Comput Biol 11:e1004189.

Hasegawa DK, Turnbull MW (2014) Recent findings in evolution and function of insect innexins. FEBS Lett 588:1403-1410.

Herberholz J, Sen MM, Edwards DH (2004) Escape behavior and escape circuit activation in juvenile crayfish during prey-predator interactions. J Exp Biol 207:1855-1863.

Hines ML, Carnevale, NT (1997) The NEURON simulation environment. Neural Comput 9:1179-1209. 
Hines ML, Davison AP, Muller E (2009) NEURON and Python. Front Neuroinform 3:1.

Hodgkin AL, Huxley AF (1952) A quantitative description of membrane current and its application to conduction and excitation in nerve. J Physiol 117:500-544.

Hormuzdi SG, Filippov MA, Mitropoulou G, Monyer H, Bruzzone R (2004) Electrical synapses: a dynamic signaling system that shapes the activity of neuronal networks. Biochim Biophys Acta 1662:113-137.

Jenett A, Rubin GM, Ngo TT, Shepherd D, Murphy C, Dionne H, Pfeiffer BD, Cavallaro A, Hall D, Jeter J, lyer N, Fetter D, Hausenfluck JH, Peng H, Trautman ET, Svirskas RR, Myers EW, Iwinski ZR, Aso Y, DePasquale GM, et al. (2012) A GAL4-driver line resource for Drosophila neurobiology. Cell Rep 2:991-1001.

King DG, Wyman RJ (1980) Anatomy of the giant fibre pathway in Drosophila. I. Three thoracic components of the pathway. J Neurocytol 9:753-770.

Koto M, Tanouye MA, Ferrus A, Thomas JB, Wyman RJ (1981) The morphology of the cervical giant fiber neuron of Drosophila. Brain Res 221:213-217.

Nakase T, Fushiki S, Naus CC (2003) Astrocytic gap junctions composed of connexin 43 reduce apoptotic neuronal damage in cerebral ischemia. Stroke 34:1987-1993.

Olsen SR, Wilson RI (2008) Cracking neural circuits in a tiny brain: new approaches for understanding the neural circuitry of Drosophila. Trends Neurosci 31:512-520.

Phelan P, Nakagawa M, Wilkin MB, Moffat KG, O'Kane CJ, Davies JA, Bacon JP (1996) Mutations in shaking-B prevent electrical synapse formation in the Drosophila giant fiber system. J Neurosci 16:1101-1113.

Phelan P, Stebbings LA, Baines RA, Bacon JP, Davies JA, Ford C (1998) Drosophila Shaking-B protein forms gap junctions in paired Xenopus oocytes. Nature 391:181-184.

Purves D, Augustine GJ, Fitzpatrick D (2001) Neuroscience. Sunderland, MA: Sinauer Associates.

Rall W (2011) Core conductor theory and cable properties of neurons. In: Handbook of physiology, the nervous system, cellular biology of neurons, pp 39-97. Hoboken, $\mathrm{NJ}$ : Wiley.
Scott EK, Reuter JE, Luo L (2003) Small GTPase Cdc42 is required for multiple aspects of dendritic morphogenesis. J Neurosci 23: 3118-3123.

Siushansian R, Bechberger JF, Cechetto DF, Hachinski VC, Naus CC (2001) Connexin43 null mutation increases infarct size after stroke. J Comp Neurol 440:387-394.

Smith HK, Roberts IJ, Allen MJ, Connolly JB, Moffat KG, O'Kane CJ (1996) Inducible ternary control of transgene expression and cell ablation in Drosophila. Dev Genes Evol 206:14-24.

Stebbings LA, Todman MG, Phillips R, Greer CE, Tam J, Phelan P, Jacobs K, Bacon JP, Davies JA (2002) Gap junctions in Drosophila: developmental expression of the entire innexin gene family. Mech Dev 113:197-205.

Sugimura K, Satoh D, Estes P, Crews S, Uemura T (2004) Development of morphological diversity of dendrites in Drosophila by the BTB-zinc finger protein abrupt. Neuron 43:809-822.

Sun YA, Wyman RJ (1997) Neurons of the Drosophila giant fiber system: I. Dorsal longitudinal motor neurons. J Comp Neurol 387:157-166.

Sutor B, Schmolke C, Teubner B, Schirmer C, Willecke K (2000) Myelination defects and neuronal hyperexcitability in the neocortex of connexin 32-deficient mice. Cereb Cortex 10:684697.

Tanouye MA, Wyman RJ (1980) Motor outputs of giant nerve fiber in Drosophila. J Neurophysiol 44:405-421.

Trimarchi JR, Schneiderman AM (1995) Different neural pathways coordinate Drosophila flight initiations evoked by visual and olfactory stimuli. J Exp Biol 198:1099-1104.

Trimarchi JR, Murphey RK (1997) The shaking-B2 mutation disrupts electrical synapses in a flight circuit in adult Drosophila. J Neurosci $17: 4700-4710$.

Walker JA, Ghalambor CK, Griset OL, McKenney D, Reznick DN (2005) Do faster starts increase the probability of evading predators? Funct Ecol 19:808-815.

Wu CL, Shih MF, Lai JS, Yang HT, Turner GC, Chen L, Chiang AS (2011) Heterotypic gap junctions between two neurons in the Drosophila brain are critical for memory. Curr Biol 21:848-854. 